\title{
Stochastic model and equivalent ferromagnetic spin chain with alternation
}

\author{
Akira Fujii 円 \\ Physikalisches Institut \\ Universität Bonn \\ Nussallee 12, 53115 Bonn \\ GERMANY.
}

\begin{abstract}
We investigate a non-equilibrium reaction-diffusion model and equivalent ferromagnetic spin 1/2XY spin chain with alternating coupling constant. The exact energy spectrum and the $n$-point hole correlations are considered with the help of the Jordan-Wigner fermionization and the inter-particle distribution function method. Although the Hamiltonian has no explicit translational symmetry, the translational invariance is recovered after long time due to the diffusion. We see the scaling relations for the concentration and the two-point function in finite size analysis.
\end{abstract}

Keywords: Spin Hamiltonians, Stochastic Models, Finite Size Study

\footnotetext{
${ }^{1}$ Present address: Universität zu Köln, Institute für Theoretische Physik, Zülpicher Str. 77, 50937 Germany
} 
The study of the systems with reaction and diffusion has been an attracting problem. Especially, methods to determine the correlation functions and to observe the approach to the thermal equilibrium in one-dimensional models have been much developed recently. One of the recent success in this field is to obtain the exact correlation functions including the coagulation term by the so-called inter-particle distribution function (IPDF) method [1, 2]. If we appropriately tune the coupling constants, (i.e. the reaction-diffusion rates) in this approach, the master equation can be solved exactly.

On the other hand, the (partially) antiferromagnetic $X Y$ spin chain with alternating coupling constant has been investigated as a toy model to consider the spin-Peierls phase transition or the Haldane gap. The thermal equilibrium model with the alternation has been studied by some authors [3, 4]. They have obtained the energy spectrum with the mass gap proportional to the strength of the alternation, the dimer correlation functions and so on. It has been also shown that the dimerizing process lowers the ground state energy. From those results, they have concluded that the uniform antiferromagnetic $X Y$-chain is unstable with respect to the distortion.

In this paper, we investigate a thermal non-equilibrium model with alternating coupling and diffusion. It can be shown that the stochastic model and the ferromagnetic $X Y$ chain with the alternation are mapped on each other by linear transformations 2, 5]. The basic tools to study these systems in this paper are the Jordan-Wigner method and the IPDF method. By the former method, which can be used in both the ferromagnetic and (partially) antiferromagnetic regimes, we can diagonalize the Hamiltonian exactly and calculate the energy spectrum. By means of the latter, which is available only in the ferromagnetic regime of the model, we obtain the exact forms of the energy spectrum and the correlation functions. Of course, the overlapped results obtained in the ferromagnetic region by both methods are the same. Since in the ferromagnetic case, the ground state occurs when all the spins are aligned, the time evolution of the correlation functions is our main interest in this paper.

Hereafter, we investigate a spin-1/2 $X Y$-model in a magnetic field along the $z$-axis with alternation whose Hamiltonian is defined with the alternation parameter $\Delta$ and the diffusion parameter $\eta(\geq 1)$ by

$$
\begin{aligned}
H & =\sum_{i=1}^{L} \frac{1}{2}\left[1+(-1)^{i} \Delta\right] H_{i, i+1} \\
H_{i, i+1} & =-\eta\left(\eta s_{i}^{x} s_{i+1}^{x}+\frac{1}{\eta} s_{i}^{y} s_{i+1}^{y}+s_{i}^{z}+s_{i+1}^{z}-\eta-\frac{1}{\eta}\right) .
\end{aligned}
$$

We assume that the length $L$ of the spin-chain is an even integer and that periodic boundary conditions $\left(\vec{s}_{L+1}=\vec{s}_{1}\right)$ are imposed.

Let us consider the exact diagonalization of the Hamiltonian (1) by the Jordan-Wigner transformation [2, 5, 6, 7]. It should be mentioned that this method is also available in both the antiferromagnetic $(\Delta<-1)$ and ferro-antiferromagnetic coexisting $(\Delta>1)$ cases as well as in the ferromagnetic $(|\Delta|<1)$ one. Due to alternation, the unique band in the uniform model is split into two separated bands with an energy gap. Therefore, we introduce two kinds of fermions $b_{2 n-1}$ and $c_{n}$ corresponding to spins on the odd and even sites respectively. The spin operators can be represented by these fermions as

$$
\begin{aligned}
& s_{2 n-1}^{+}=b_{2 n-1}^{\dagger} \exp \left(i \pi \sum_{j=1}^{n-1} b_{2 j-1}^{\dagger} b_{2 j-1}+i \pi \sum_{j=1}^{n-1} c_{2 j}^{\dagger} c_{2 j}\right), s_{2 n-1}^{-}=\left(s_{2 n-1}^{+}\right)^{\dagger}, \\
& s_{2 n}^{+}=c_{2 n}^{\dagger} \exp \left(i \pi \sum_{j=1}^{n} b_{2 j-1}^{\dagger} b_{2 j-1}+i \pi \sum_{j=1}^{n-1} c_{2 j}^{\dagger} c_{2 j}\right), s_{2 n}^{-}=\left(s_{2 n}^{+}\right)^{\dagger},
\end{aligned}
$$


where the integer $n$ runs from 1 to $L / 2$. In terms of the fermions $b$ and $c$, the boundary conditions are given by $b_{L+i}= \pm b_{i}$ and $c_{L+i}= \pm c_{i}$ depending on whether $\mathcal{N}=\sum_{j=1}^{L / 2}\left(b_{2 j-1}^{\dagger} b_{2 j-1}+c_{2 j}^{\dagger} c_{2 j}\right)$ is odd or even. Performing the Fourier transformation

$$
b_{2 n-1}=\sqrt{\frac{2}{L}} \sum_{k} e^{-\frac{2 \pi i k}{L}(2 n-1)} \bar{b}_{k}, \quad c_{2 n}=\sqrt{\frac{2}{L}} \sum_{k} e^{-\frac{2 \pi i k}{L} \cdot 2 n} \bar{c}_{k},
$$

and the Bogoliubov transformation

$$
\left(\tilde{b}_{k}, \tilde{b}^{k}, \tilde{c}_{k}, \tilde{c}^{k}\right)=\left(\bar{b}_{k}, \bar{b}_{-k}^{\dagger}, \bar{c}_{k}, \bar{c}_{-k}^{\dagger}\right) S_{k}
$$

where $S_{k}$ is a certain $(4 \times 4)$-matrix, we can rewrite the Hamiltonian (1) as

$$
\begin{aligned}
& H=\sum_{k} \tilde{H}_{k}, \\
& \tilde{H}_{k}+\tilde{H}_{\frac{L}{2}-k}=\lambda_{k}^{(+)}\left(\tilde{b}_{k}^{\dagger} \tilde{b}_{k}-\tilde{b}^{k \dagger} \tilde{b}^{k}\right)+\lambda_{k}^{(-)}\left(\tilde{c}_{k}^{\dagger} \tilde{c}_{k}-\tilde{c}^{k \dagger} \tilde{c}^{k}\right) .
\end{aligned}
$$

The energy eigenvalues $\lambda_{k}^{( \pm)}$are given by

$$
\lambda_{k}^{( \pm)}=-\left(\eta^{2}+1\right) \pm 2 \eta \sqrt{\cos ^{2}\left(\frac{2 \pi k}{L}\right)+\Delta^{2}\left[\sin ^{2}\left(\frac{2 \pi k}{L}\right)+\left(\frac{\eta-\eta^{-1}}{2}\right)^{2}\right]}
$$

where the value of momentum $k$ depends on $\mathcal{N}$ as

$$
\begin{aligned}
k & =0,1,2, \cdots, \frac{L}{2}-1, \quad \text { for } \mathcal{N}=\text { odd }, \\
k & =\frac{1}{2}, \frac{3}{2}, \cdots, \frac{L}{2}-\frac{1}{2}, \quad \text { for } \mathcal{N}=\text { even } .
\end{aligned}
$$

Therefore, the ground state is given by the half-filled state in this basis like

$$
\left(\prod_{k} \tilde{b}_{k}^{\dagger}\right)\left(\prod_{k} \tilde{c}_{k}^{\dagger}\right)|\widetilde{v a c}\rangle \quad \text { with } \quad \tilde{b}_{k}|\widetilde{v a c}\rangle=\tilde{b}^{k}|\widetilde{v a c}\rangle=\tilde{c}_{k}|\widetilde{v a c}\rangle=\tilde{c}^{k}|\widetilde{v a c}\rangle=0
$$

and the energy spectrum has a gap $2 \eta \Delta\left(\eta+\eta^{-1}\right)$ at $2 \pi k / L \sim \pi / 2$ provided $\Delta \neq 0$ as is shown

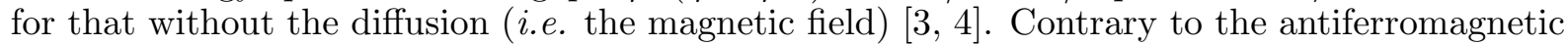
case, the energy gap does not appear near the Fermi surface in the ferromagnetic case. It follows that the ground state energy remains a monotonically increasing function of the alternation parameter $\Delta$ even if we add the elastic energy of distortion to the Hamiltonian. It means that the instability due to the dimerization cannot be observed in the ferromagnetic case.

On the other hand, as is shown in [2, 5], the spin Hamiltonian (1) can be mapped onto a stochastic model including coagulation and decoagulation processes by regarding the spin-up and spin-down state as sites occupied by a particle (denoted $A$ ) and empty (denoted $\phi$ ), respectively. If the $i$-th site is occupied (empty), we label $\sigma_{i}=1(0)$. The stochastic model to be considered hereafter is defined by the transition rates $w_{\alpha \beta}(\mu, \nu)\left(\alpha, \beta, \mu, \nu \in \mathbf{Z}_{2}\right)$ and arbitrary positive constants $a_{ \pm}$. Only the following processes are allowed.

1. Diffusion

$A+\phi \leftrightarrow \phi+A$ at the rate $a_{ \pm} w_{11}(01)=a_{ \pm} w_{11}(10)$ 
2. Coagulation

$A+A \rightarrow A+\phi$ and $A+A \rightarrow \phi+A$ at the same rate $a_{ \pm} w_{01}(10)=a_{ \pm} w_{10}(01)$.

3. Decoagulation

$A+\phi \rightarrow A+A$ and $\phi+A \rightarrow A+A$ at the same rate $a_{ \pm} w_{01}(11)=a_{ \pm} w_{10}(11)$.

The above rates should be interpreted as below. For example, let us assume that the $i$-th and $(i+1)$-th sites are occupied and empty, respectively, and that $i$ is an even (odd) integer. After an infinitesimal time interval $d t$, the sites will be occupied by $\phi$ and $A$, respectively, at the rate $a_{+} w_{10}(01) d t\left(a_{-} w_{10}(01) d t\right)$. We restrict the rates further as

$$
\begin{aligned}
& w_{11}(10)=w_{11}(01)=w_{01}(10)=w_{10}(01)=1, \\
& w_{01}(11)=w_{10}(11)=\eta^{2}-1,
\end{aligned}
$$

which makes it possible to solve the model exactly by the IPDF method as will be shown later. Because we can fix the normalization of $a_{+}$and $a_{-}$as $a_{+}+a_{-}=1$ by rescaling time, we put $a_{ \pm}=(1 \pm \Delta) / 2$ with the same $\Delta$ as in (1). The basic quantity in the stochastic model should be the probability distribution function $P(\underline{\sigma} ; t)$, which is the probability to find the system in the configuration $\underline{\sigma}=\left(\sigma_{1}, \cdots, \sigma_{L}\right)$ at time $t$. Defining the Hamiltonian by

$$
\begin{aligned}
\hat{H} & =\sum_{j=1}^{L} a_{(-)^{j}} \hat{H}_{j, j+1} \text { with } a_{(-)^{\text {odd }}}=a_{-}, a_{(-) \text {even }}=a_{+}, \\
\left(\hat{H}_{i, i+1}\right)_{\sigma_{i} \sigma_{i+1}}^{\rho_{i} \rho_{i+1}} & =\left\{\begin{array}{lll}
w_{\sigma_{i}-\rho_{i}, \sigma_{i+1}-\rho_{i+1}}\left(\sigma_{i}, \sigma_{i+1}\right), & \text { if } \quad\left(\rho_{i}, \rho_{i+1}\right) \neq\left(\sigma_{i}, \sigma_{i+1}\right) \\
-\sum_{\alpha \neq 0, \beta \neq 0} w_{\alpha \beta}\left(\sigma_{i}, \sigma_{i+1}\right), & \text { if } \quad\left(\rho_{i}, \rho_{i+1}\right)=\left(\sigma_{i}, \sigma_{i+1}\right)
\end{array}\right.
\end{aligned}
$$

with periodic boundary conditions $i \equiv i+L$, we can write the master equation of the probability distribution function in terms of a Schrödinger equation with imaginary time

$$
\begin{aligned}
\frac{\partial}{\partial t} P\left(\sigma_{1}, \cdots, \sigma_{L} ; t\right)= & -\sum_{j=1}^{L} \sum_{\rho_{j}, \rho_{j+1}=1,0} a_{(-)^{j}}\left(\hat{H}_{j, j+1}\right)_{\sigma_{j} \sigma_{j+1}}^{\rho_{j} \rho_{j+1}} \times \\
& \times P\left(\sigma_{1}, \cdots, \sigma_{j-1}, \rho_{j}, \rho_{j+1}, \sigma_{j+2}, \cdots, \sigma_{L} ; t\right) .
\end{aligned}
$$

The Hamiltonian above given can be transformed into that of the spin system (11) by the same mapping proceeded by [2, 5]. Performing a similarity transformation $\hat{H}_{1}=U^{-1} \hat{H} U$ with the matrix

$$
U=\left(\begin{array}{cc}
\sqrt{\eta^{2}-1} & 0 \\
0 & 1
\end{array}\right) \otimes \cdots \otimes\left(\begin{array}{cc}
\sqrt{\eta^{2}-1} & 0 \\
0 & 1
\end{array}\right),
$$

and a rotational transformation $H=R^{-1} \hat{H}_{1} R$ with

$$
R=\exp \left(i \theta s_{1}^{y}\right) \otimes \cdots \otimes \exp \left(i \theta s_{L}^{y}\right) \quad\left(\tan \theta=\sqrt{\eta^{2}-1}\right)
$$

we obtain the spin Hamiltonian (1).

To calculate the correlation function from the master equation (12), we use the IPDF method [2, 5, 8] - [10]. For simplicity, we assume a Gaussian initial condition for general $N$ and independent of $M$ as

$$
\sum_{\underline{\sigma}} P(\underline{\sigma} ; t=0) \delta_{\sigma_{M}, 0} \cdots \delta_{\sigma_{M+N-1}, 0}=p^{N}
$$


where $p(0 \leq p \leq 1)$ is the probability of a site to be empty. If we define the "hole length probability" (HLP) $\Omega(m ; t)$, which is the probability to find a string of empty sites with the length $m$ at time $t$, by

$$
\begin{aligned}
\Omega(2 n+1 ; t) & =\sum_{\underline{\sigma}} P(\underline{\sigma} ; t) \delta_{\sigma_{M}, 0} \cdots \delta_{\sigma_{M+2 n}, 0} \\
\Omega^{( \pm)}(2 n ; t) & =\sum_{\underline{\sigma}} P(\underline{\sigma} ; t) \delta_{\sigma_{M}, 0} \cdots \delta_{\sigma_{M+2 n-1}, 0}, \quad(M=\text { even/odd }),
\end{aligned}
$$

we can easily verify that the above $\Omega$ 's do not depend on the starting site $M$ explicitly because of the translational invariant initial condition (15). Note that there are two kinds of the HLP's $\Omega^{( \pm)}$for even hole-lengths, depending on whether the starting point $M$ is even or odd. With these preparations, we can rewrite the master equation simply as

$$
\begin{array}{rlrl}
\frac{\partial}{\partial t} \Omega(2 n-1 ; t) & =\eta^{2}\left[a_{+} \Omega^{(+)}(2 n ; t)+a_{-} \Omega^{(-)}(2 n ; t)\right]-\left(1+\eta^{2}\right) \Omega(2 n-1) \\
& +\left[a_{+} \Omega^{(+)}(2 n-2 ; t)+a_{-} \Omega^{(-)}(2 n-2 ; t)\right] \quad & \text { for } 1 \leq n \leq \frac{L}{2}, \\
\frac{\partial}{\partial t} \Omega^{( \pm)}(2 n ; t) & =2 a_{\mp}\left[\eta^{2} \Omega(2 n-1 ; t)-\left(1+\eta^{2}\right) \Omega^{( \pm)}(2 n ; t)\right. & & \Omega(2 n+1 ; t)] \\
& & \text { for } 1 \leq n \leq \frac{L}{2}-1 .
\end{array}
$$

The solution of Eq.(17) is given by

$$
\begin{aligned}
\Omega(2 n-1 ; t)= & \sum_{l=1}^{L / 2-1} \sum_{s= \pm} A_{l}^{(s)} \eta^{-(2 n-1)} e^{\lambda_{l}^{(s)} t} \sin \left(\frac{\pi(2 n-1)}{L} l\right)+ \\
& +B_{2 n-1} e^{-\left(\eta^{2}+1\right) t}+\psi(2 n-1), \\
\Omega^{( \pm)}(2 n ; t)= & \sum_{l=1}^{L / 2-1} \sum_{s= \pm} A_{l}^{(s)} \eta^{-(2 n+1)} \frac{\lambda_{l}^{(s)}+2 a_{ \pm}\left(\eta^{2}+1\right)}{4 a_{ \pm}} e^{\lambda_{l}^{(s)}} t \frac{\sin (2 \pi n l / L)}{\cos (\pi l / L)}+ \\
& +\frac{1-a_{ \pm}}{1-2 a_{ \pm}} \frac{2}{\eta^{2}+1}\left(B_{2 n-1}+\eta^{2} B_{2 n+1}\right) e^{-\left(\eta^{2}+1\right) t}+\psi(2 n) .
\end{aligned}
$$

In the above expression, $A_{l}^{( \pm)}$and $B_{2 n-1}$ are complicated coefficients depending on $L, \Delta, p, \eta$ and $l$ and the zero-mode function is

$$
\psi(m)=\frac{1}{1-\eta^{-2 L}}\left[\left(1-p^{L}\right) \eta^{-2 m}+p^{L}-\eta^{-2 L}\right] .
$$

The energy spectrum $\lambda_{l}^{( \pm)}$takes the same form as that in (16).

With the above solution, we can investigate the finite size scaling of physical quantities. From now on, we set $\eta=1$, i.e. the massless regime. First, we consider the finite size correction of the concentration $c(t)=1-\Omega(1 ; t)$ [5]. It is not difficult to ensure that we can fix a parameter $z=2\left(1-\Delta^{2}\right) t / L^{2}$ finite in the scaling limit $L \rightarrow \infty$ and $t \rightarrow \infty$. After performing a modular transformation $z \rightarrow-1 / z$, the asymptotic form of the concentration reads

$$
\begin{aligned}
c(t) & \sim \sqrt{\frac{1}{2 \pi\left(1-\Delta^{2}\right) t}} \times \\
& \times\left(1-\frac{1}{32\left(1-\Delta^{2}\right) t}\left[\frac{1+6 p+p^{2}}{(1-p)^{2}}+\Delta \frac{3-2 p+3 p^{2}}{(1+p)^{2}}\right]\right)+O\left(t^{-5 / 2}\right) .
\end{aligned}
$$


The finite size analysis for the two hole probability $\Omega^{( \pm)}(2 ; t)$, which is identified with the probability to find a dimer on a link for $a_{ \pm}$, can be done similarly. Due to alternation, $\Omega^{(+)}(2 ; t)$ and $\Omega^{(-)}(2 ; t)$ do not coincide for general $t$ in spite of the initial condition, $\Omega^{(+)}(2 ; 0)=\Omega^{(-)}(2 ; 0)=p^{2}$. However, because of the diffusion and coagulation, we will observe $\Omega^{(+)}(2 ; \infty)=\Omega^{(-)}(2 ; \infty)=1$, which indicates the recovery of the translational invariance. For example, let us consider the ratio of the two probabilities $\Omega^{(+)}(2 ; t)$ and $\Omega^{(-)}(2 ; t)$. After a lengthy calculation, its finite size scaling is shown to be

$$
\frac{\Omega^{(-)}(2 ; t)}{\Omega^{(+)}(2 ; t)} \sim 1+\frac{\Delta}{\sqrt{2 \pi}}\left(\left(1-\Delta^{2}\right) t\right)^{-3 / 2}+O\left(t^{-5 / 2}\right) .
$$

As we have seen, both the Jordan-Wigner method and the IPDF approach are available in the XY model with diffusion and alternation. An energy gap proportional to the strength of the alternation is observed. We have also seen that the translational invariance is recovered by the diffusion beginning with a translational invariant initial condition. This recovery, e.g. $\Omega^{(-)}(2 ; t) / \Omega^{(+)}(2 ; t)$, is scaled by the same variable $z$ as that of the concentration. We have obtained the exponents of the concentration and the two hole probabilities in the scaling limit $L, t \rightarrow \infty$.

We can imagine some extension in this field. First, the coupling constants do not have to change on every other link in our approaches. That is, it is possible to change the coupling constant on every third (fourth and so on) site without loss of solvability. In particular, it would be interesting to consider the relation to the model with randomly changing coupling constants [11. Secondly, contrary to the IPDF method, the Jordan-Wigner method can be also applied for the (partially) antiferromagnetic alternating model. Although we did not succeed in finding the corresponding initial condition in the Jordan-Wigner approach so far, we expect that a parallel discussion can be done.

\section{Acknowledgements}

The author would like to thank J. Gruneberg, T. Heinzel, Y.U. Lee, R. Raupach and V. Rittenberg for reading the manuscript, helpful comments and suggestion. The discussions with J. Suzuki and the theory group in the University of Bonn are also acknowledged. This work is financially supported by the AvH foundation.

\section{References}

[1] Kadanoff L P and Swift J 1968 Phys. Rev. 165310

[2] Alcaraz F C, Droz M, Henkel M and Rittenberg V 1994 Ann. Phys. (NY) 230250

[3] Pincus P 1971 Solid State Comm. 91971

[4] Okamoto K 1988 J. Phys. Soc. Jpn. 57 2947; 1990 J. Phys. Soc. Jpn. 59 4286; Saika Y and Okamoto K 1995 "Dimer correlation in spin-1/2 alternative XY chain" e-print: cond-mat/9510114

[5] Hinrichsen H, Krebs K, Pfannmüller M and Wehefritz B 1995 J. Stat. Phys. 781429

[6] Barouch E, McCoy B M and Dresden M 1970 Phys. Rev. A2 1075

[7] Hinrichsen H 1994 J. Phys. A27 1121 
[8] ben-Avraham D, Burschka M A and Doering C R 1990 J. Stat. Phys. 60695

[9] Peschel I, Rittenberg V and Schultze U 1994 Nucl. Phys. B430 633;

Schütz G 1995 J. Stat. Phys. 79243

[10] Henkel M and Schütz G 1994 Physica A206 187;

Lushnikov A A 1986 Sov. Phys. JETP 64 811; 1987Phys. Lett. A120 135

[11] Evans M R 1996 Europhys. Lett. 3613 